\title{
Behaviour of Torsionally Coupled Buildings with Tuned Mass Dampers
}

\author{
Arshid Bashir \\ M.Tech (Structures \& Foundation), Alfalah University, Dhauj, Faridabad \\ Mirza Aamir Baig \\ Assistant Professor, Alfalah University, Dhauj, Faridabad
}

\begin{abstract}
Elevated structure structures are both a need and a matter of advancement and pride for auxiliary architects. Powers of nature as quakes and typhoons begins playing merciless games with the structures, higher the structure goes, and higher it pulls in the powers and fury of nature as seismic power.

Seismic power, prevalently being an inactivity power relies upon the mass of the structure causing necessity of heavier segments as mass increments. Also, these substantial areas further increment the mass of the structure prompting significantly heavier seismic powers. Basic fashioners are met with gigantic test to adjust these opposing physical wonders to make the structure safe. The structure no more can bear to be inflexible.

In late year, it is basic practice to introduce vibration control gadgets on structures to relieve their dynamic reaction brought about by various components, for the most part because of wind and tremor excitations. Among these gadgets, tuned mass dampers (TMD) have been broadly utilized in working to moderate unique reactions of structures. As the auxiliary unpredictability of structures increment, their reaction to such excitations may turn out to be more inclined to torsional movement. Torsional movement actuated by such excitations can be smothered by using TMDs. The exhibition of TMD at various positions are significant for torsionally coupled structures. In this examination, the presentation of bi-directional tuned mass dampers (BTMD) in lessening the torsional reaction of a structure under bi-directional seismic tremor excitations was contemplated and assessed.
\end{abstract}

Keywords: Tuned Mass Dampers, TMD, BTMD, ATMS, Seismic Analysis, Torsionally Coupled Buildings, Irregularities

\section{INTRODUCTION}

A significant aspect of the vitality of ground seismic waves arriving at the base of building is diminished because of avoidance of seismic waves inside the earth, however the reaming part has destroying impact. When the seismic waves enter the dinner structure of a structure or some other structure; the structure or structure has either to oppose without anyone else by its unique plan or helped by different methods to oppose the impact of the vibration brought about by tremors. Improving the structure's seismic exhibition utilizing vibration control gadgets is among the procedures utilized. The fundamental thought of most damping gadgets is to check the info vitality by their own energy.

By and large basic vibration controlling framework can be separated into four kinds for example Dynamic control frameworks, uninvolved control frameworks, semi dynamic control frameworks and Hybrid control frameworks as portrayed in the following segments. These frameworks use vibration safeguards (gadgets) for their executions. Lately effective a down to earth vibration control has been accomplished by the application or dynamic vibration safeguards. Seismic vibration control gadgets can be latent, dynamic, semi dynamic and mixture gadgets. The gadgets are utilized to improve vibration execution by disseminating the wave vitality inside a superstructure; by scattering the wave vitality between a more extensive scope of frequencies; and by retaining the resounding parts of the entire wave frequencies.

\subsection{Tuned mass dampers}

Tuned mass dampers (TMD) have been broadly utilized for vibration control in mechanical designing frameworks. Lately, TMD hypothesis has been embraced to lessen vibration of tall structures and other structural building structures. The recurrence of the damper is tuned to a specific auxiliary recurrence so when that recurrence is energized, the damper will reverberate out of stage with the basic movement. The component for alleviating basic vibrating utilizing a TMD includes moving the vibration vitality from the structure into the TMD, which dispersed this vitality through the damping impact. TMDs work by giving extra damping to the structure. TMD gives recurrence subordinate hysteresis which increments damping in the edge structure appended to it so as to diminish its movement. TMDs are viable in controlling vibration of structure exposed to long length, tight band excitations

Through escalated innovative work as of late, TMD are acknowledged as a powerful vibration control gadget for both new and existing structures; to risk their dependability against winds, quake, and vibrations because of human exercises. TMDs are gadgets comprising of a mass, a spring, and a gooey damper that is appended to a structure so as to lessen the dynamic reaction of the structure so as to decrease dynamic reaction of the structure as characterized in the past part. The TMD idea was first applied by Frahm in 1909 to decrease the moving movement of boats just as boat body vibrations. The basic model utilized by Frahm had just safeguard mass suspended by undamped spring and had the option to set the vibration adequacy of the fundamental framework 
to zero for a solitary recurrence. A hypothesis for the TMD was introduced later in the paper by Ormandy and Den Hartog (1940). The underlying hypothesis was pertinent for an undamped SDOF framework exposed to a sinusoidal power excitation.

\subsection{Working principles of TMD}

A TMD parts the characteristic recurrence of the essential framework into a lower recurrence (f1) and higher recurrence (f2), the recurrence reaction chart of SIDOF framework is plotted for a given mass apportion alongside the ideal tuning proportion y pick and ideal damping proportion $\xi$ decide on $\xi=0$ or $\xi=\infty$, the pinnacle reaction is vast. When $\xi=0$, the two massed carry on freely with the two pinnacles showing up on the recurrence reaction diagrams watching out for endlessness. When $\xi=\infty$, the dampers are intertwined (bolted) with the structure and the framework acts as a SDOF with mass as the entirety of the TMD and the structure. At the point when the damping proportion isn't at its ideal worth, for example, $\xi=0.7 \xi$ select tow pinnacles of various tallness show up. At the point when both the tuning proportion damping proportion are at their ideal qualities, two pinnacles of equivalent tallness happen with the most reduced pinnacle reaction. So, planning a TMD having this equivalent reaction tops. It is imperative to appropriately plan the tuned mass damper to accomplish its most extreme presentation. The presentation of a TMD is generally assessed regarding the powerful damping added to the structure by the TMD. The compelling damping $\xi$ eff.is characterized as a solitary hosing boundary in a SDOF structure that will give a similar presentation as though the structure is joined with a TMD (McNamara, 1977)

\subsubsection{Torsionally coupled building installed with TMDs}

Structure for which the focuses of mass and inflexibility don't concur are alluded to as awry or torsionally coupled structure and separation between these two focuses is called whimsy. In the event that focuses of mass and inflexibility of the floor these two focuses is called whimsy. In the event that focuses of mass and unbending nature of the floor stomachs lie along a similar vertical pivot, a level segment of ground vibration will incorporate just horizontal or interpretation movement of the structure on which it is acting. Then again, if the focuses of mass and unbending nature to don't harmonize, an even part of excitation will by and large actuate both horizontal segments of movements and a rotational segment about a vertical hub. Torsional activities by likewise be actuate in symmetric structures significantly under a simply interpretation segment of ground excitation, if all purposes of the base of the structure are not energized all the while in view of the limited speed of spread of the ground excitation, Kau (1974). Deviated building will encounter a coupled torsional translational movement in any event, when they are energized by an absolutely translational movement of the ground. The torsional segment of the reaction may contribute altogether to the general reaction of the structure, especially when the uncoupled torsional and translational frequencies of the framework are near one another $(\Omega-1)$

The torsional reaction conduct of a structure relies upon the boundary called uncoupled recurrence proportion which is the proportion of the two uncoupled characteristic frequencies of the structure $(\Omega)$ RaminTabatabaei (2011) given by,

$\Omega=\dot{\omega} 0$

\section{$\Omega$ interpretation}

Where $\omega$ interpretation and $\omega 0$ are an uncoupled interpretation and torsional frequencies, individually $\Omega$ interpretation can be in $\mathrm{x}$ or $\mathrm{y}$ course. The estimations of the proportion $(\Omega)$ can be acquired by changing the auxiliary boundaries for example firmness of the structure, and separation of the opposing components from the focal point of mass.

In the event that $\Omega>1$, the structure is characterized as torsionally solid and its reaction is principally translational

On the off chance that $\Omega>1$, the structure is emphatically torsionally coupled, so that the translational and the rotational reactions happen simultaneously for any excitation.

In this investigation, the reaction of the apparent multitude of three cases with and without BTMD are considered alongside various flightiness proportions under bi-directional quake excitations.

Truly leaving building for the most part have countless level of opportunity, and are really awry, even with a balanced arrangement. Such a structure will go through Lateral also torsional vibrations all the while under unadulterated translational excitations.

In ongoing explores works, the utilization of TMDs in controlling the torsional reaction structures are being researched jangidan Datta (1997) examined the viability of MTMD on diminishing the reaction of torsionally coupled framework, which comprises of two level of opportunity structure with rotational and translational movement under fixed irregular excitation.

One of the most significant contemplations in the plan of TMDs is the situation of the TMDs on the structure considered particularly for torsional structures. The issue of distinguishing the ideal area of the TMDs in order to expand the decrease of the 
structure seismic reaction have been concentrates by various examination for example Lin et al (2008), Fu and Johnsen (2011) Lin et al (1995) in their investigation of vibration control ID of seismically energized MDOF structure and found that for planer structure the floor relating to the tip of controlled mode shape will be the ideal area of PTMD additionally Ueng et al. (1999) contemplates the vibration control viability of PTMD for lessening plan the seismic reaction or torsionally coupled structures. Considering the functional plan issues for example the ideal area for the establishment; planer position; and development course of PTMDs; their investigation reasoned that the more noteworthy the separation between the PTMD Wu (2001) built up a consecutive technique to decide the quantity of modes to be controlled and the ideal conveyance of TMD framework comprising of dampers put on each floor, rather than dense in one or in a couple of areas, as utilized in conventional TMDs, and damping coefficients. |I this examination the successful situation of BTMD on the arrangement of q Single and five story building.

\section{MODEL CONSIDERATION \& ANALYSIS}

Design Characteristics: - The following design characteristics are considered for Multi-storey rigid jointed frames

Table 1: Design Data of RCC Frame Structures

\begin{tabular}{|l|l|l|}
\hline S.No & Particulars & Dimension/Size/Value \\
\hline 1. & Model & $2 \mathrm{~B}+\mathrm{G}+10$ \\
\hline 2. & Seismic Zones & $\mathrm{IV}$ \\
\hline 3. & Floor height & $3 \mathrm{~m}$ \\
\hline 4. & Basement & $3 \mathrm{~m}$ \\
\hline 5. & Building height & $39 \mathrm{~m}$ \\
\hline 6. & Plan size & $20 \mathrm{mx} 12 \mathrm{~m}$ \\
\hline 12. & Earthquake load & As per IS-1893-2002 \\
\hline & Type of soil & Type -II, Medium soil as per IS-1893 \\
\hline 13. & Live load & $2 \mathrm{kN} / \mathrm{m}^{2}$ \\
\hline 16. & Zone factor Z & \\
\hline
\end{tabular}

\subsection{Seismic Load}

As per IS: 1893, Noida is located in Seismic Zone IV.

Design base shear, $\mathrm{V}=\mathrm{Z}$ I W S $\mathrm{a} / 2 \mathrm{Rg}$

The values of the salient coefficients are tabulated below:

Table 2 Seismic parameters

\begin{tabular}{|l|l|l|}
\hline Description & Value \\
\hline Seismic Factor for Zone : IV & 0.24 & Reference \\
\hline Structure Importance coefficient, I. & 1.0 & \\
& & \\
\hline Response reduction factor, R & 5.00 & IS-1893 \\
\hline Damping & $5 \%$ & IS-1893 \\
\hline Time period & Variable & IS-1893 \\
\hline
\end{tabular}

Wind Load

The wind velocity at Noida is $47 \mathrm{~m} / \mathrm{s}$. The other parameter of wind load as per IS: 875 (Part-3) is summarized below:

Table 3: Wind parameters

\begin{tabular}{|l|l|l|l|}
\hline S1. & Description & Value & Reference \\
\hline 01 & Terrain category. & 3 & IS-875 \\
\hline 02 & Class of structure. & C & IS-875 \\
\hline 03 & Probability factor, $\mathrm{k}_{1}$. & 1.0 & IS-875 \\
\hline 04 & Terrain, height and structure size factor, $\mathrm{k}_{2}$. & As/Height & IS-875 \\
\hline 05 & Topography factor, $\mathrm{k}_{3}$. & 1.0 & IS-875 \\
\hline
\end{tabular}

\subsection{Torsionally Coupled Buildings}

In basic words, structures whose focal point of mass don't correspond with its focal point of unbending nature are called torsionally coupled structures. One aspect of the structure initiates twists on other aspect of the structure. In this way, regardless of what be the bearing of seismic tremor, the structure will consistently vibrate prevalently in twist.

In the above building, the $\mathrm{CM}$ is not the same as the CR. Along these lines, extra second makes the structure pivot about its vertical hub when followed up on any sidelong burden.

Here an endeavour has made to comprehend the vibration conduct of torsionally coupled structures under seismic burdens. A straightforward 10 story structure has been taken for study. A similar structure was breaking down for seismic burdens, static and 
dynamic. Time history investigation has additionally been completed to comprehend its vibration conduct. The structure considered is demonstrated as follows.

\subsubsection{Torsionally Coupled without TMD (Type A)}
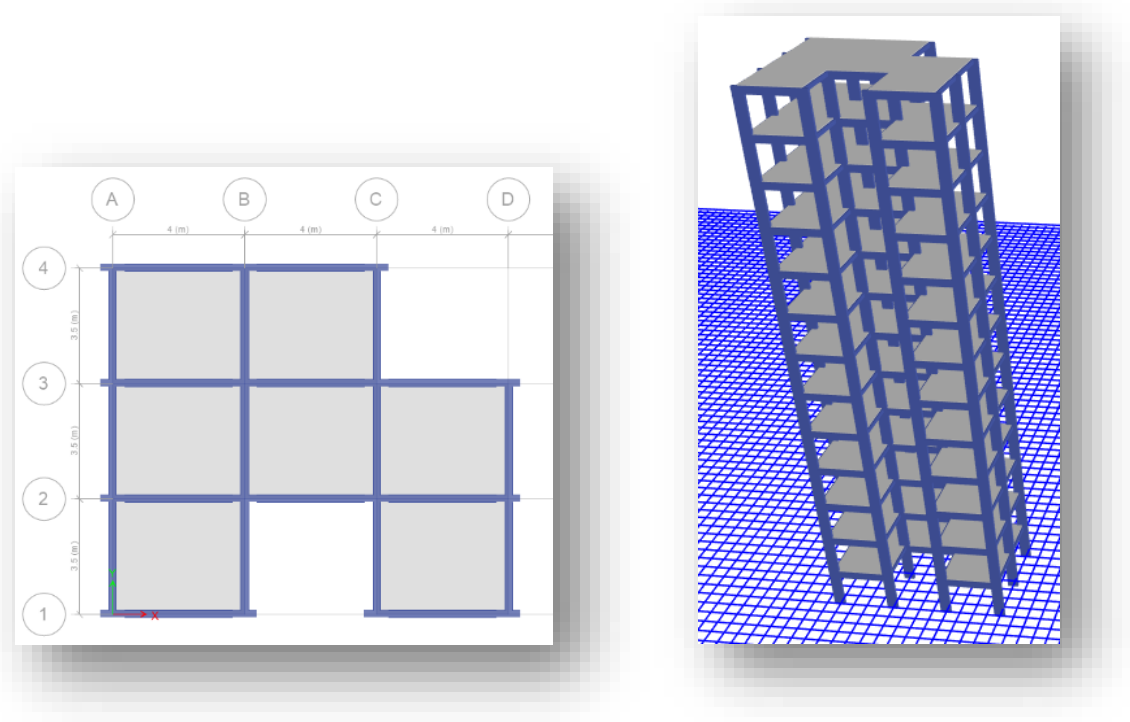

Storey Displacement for Type A

The storey displacements are plotted below for EQX, EQY, SPECX \& SPECY.

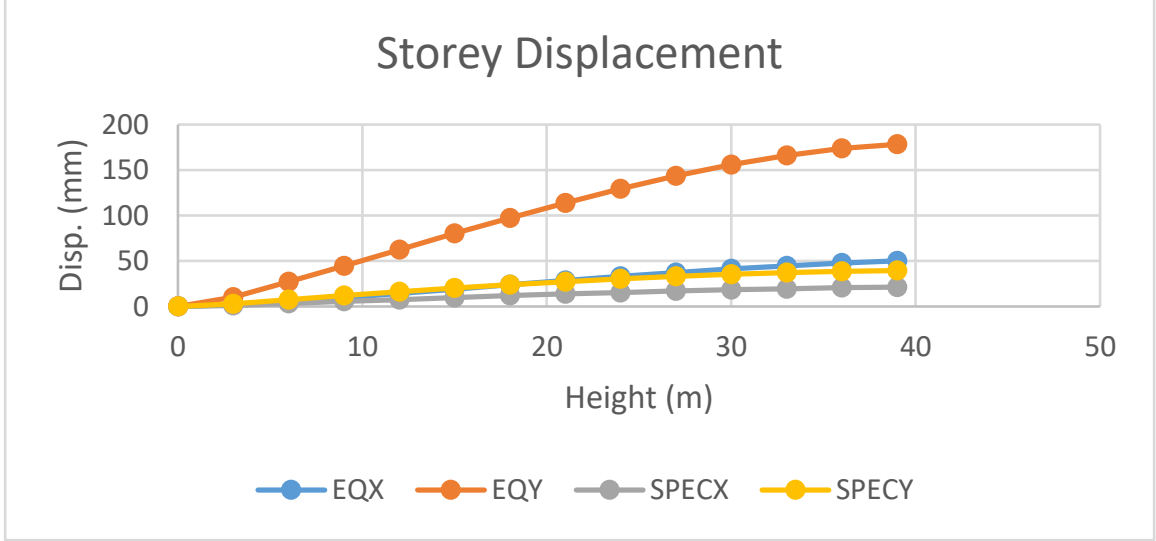

Storey Shear for Type A

The storey shears are plotted below for EQX, EQY, SPECX \& SPECY.

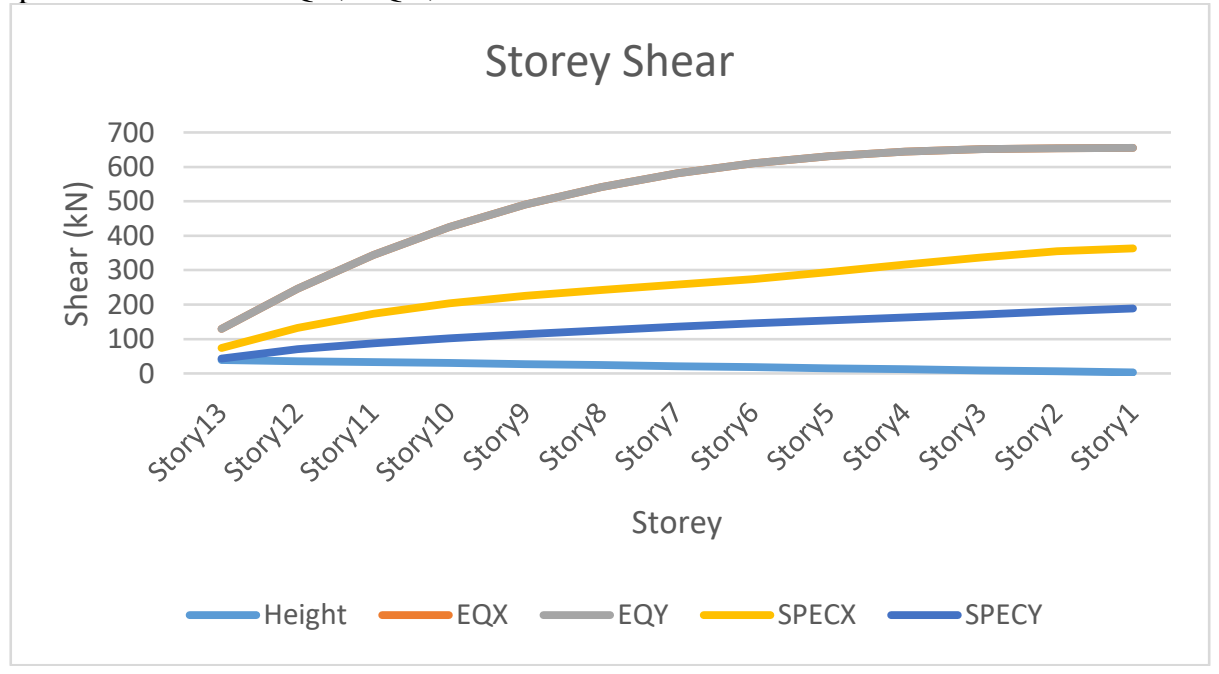


Torsionally Coupled with TMD (Type B)

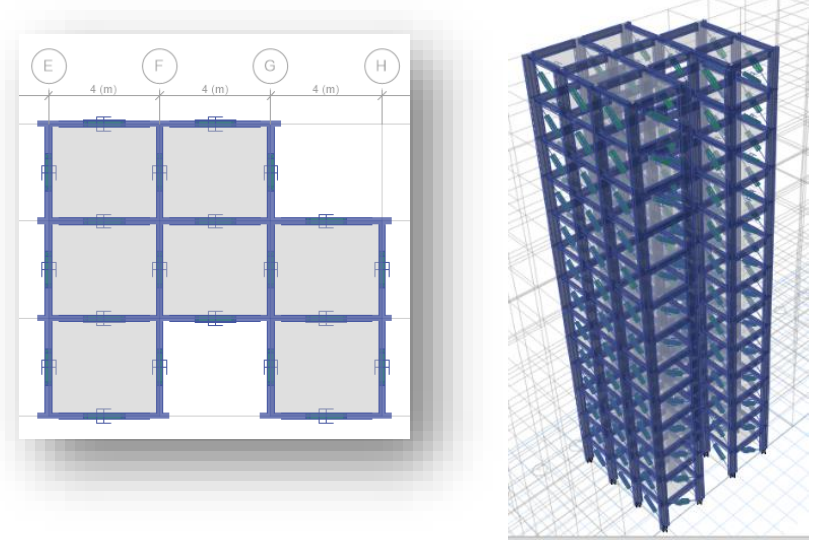

Storey Displacement for Type B

The storey displacements are plotted below for EQX, EQY, SPECX \& SPECY.

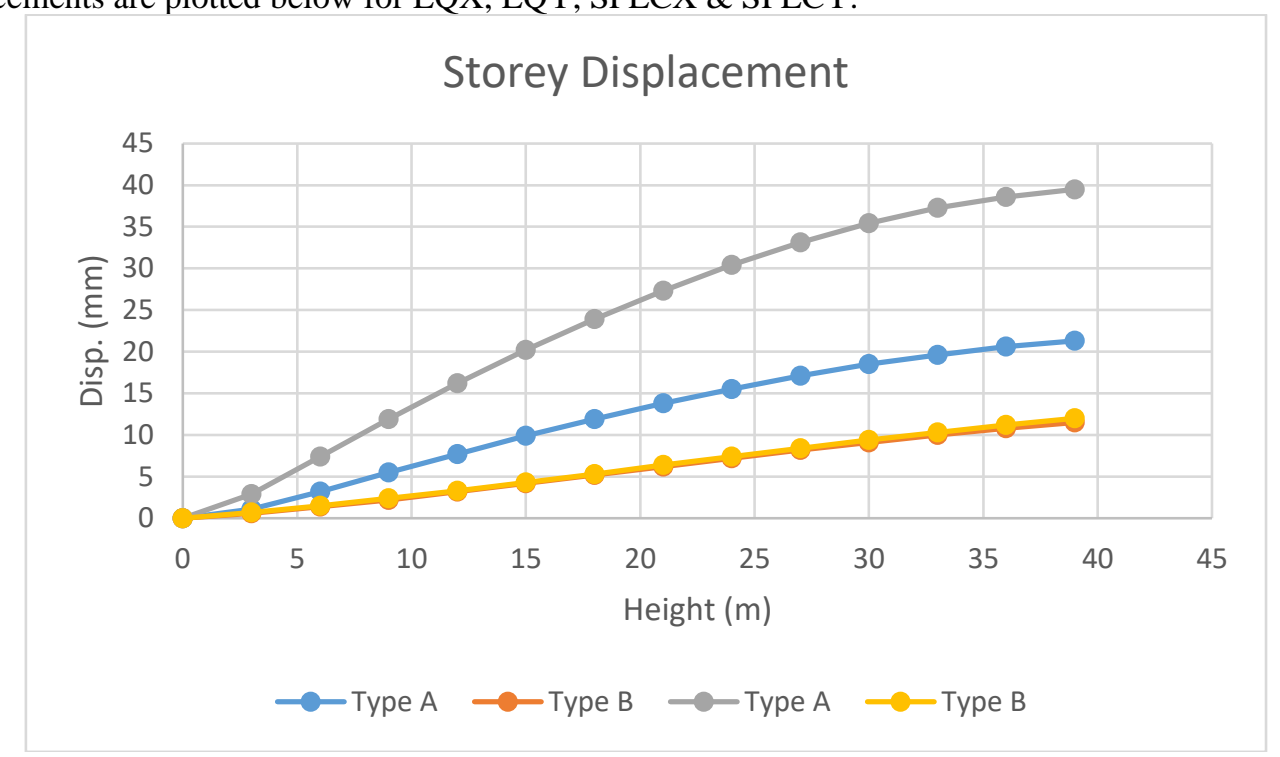

As expected the displacements are much reduced compared to Type A.

Storey Shear for Type B

The storey shears are tabulated below for EQX, EQY, SPECX \& SPECY.

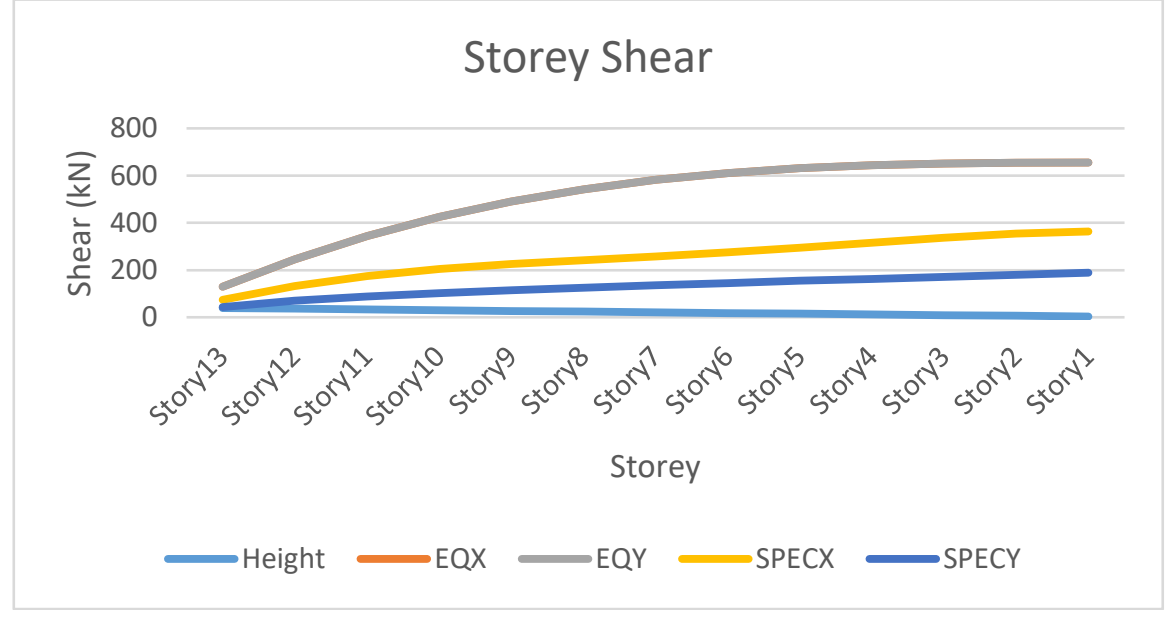

Here we observe the base shear is same as Type A, as the weight of the two structures are same. Thus the base shears are same. 
Let's compare the storey displacements for both the structures for EQX and EQY.

\section{Storey Displacement}

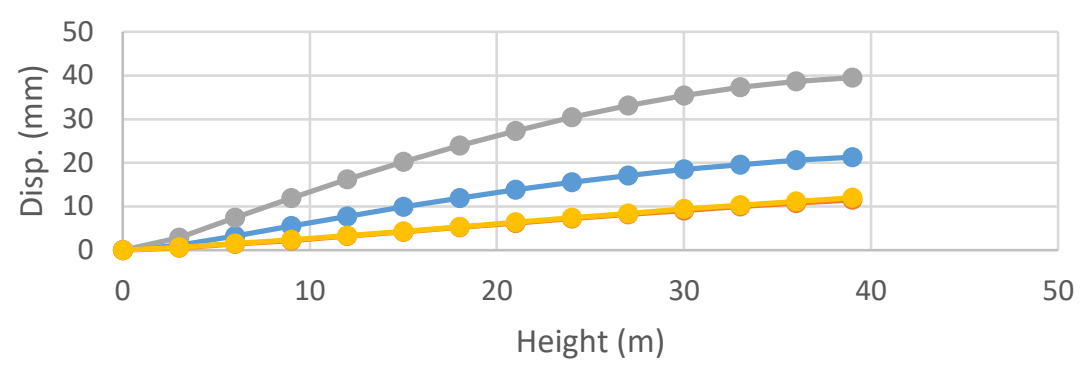

-Type A $\longrightarrow$-Type B $\longrightarrow$-Type A $\multimap$-Type B

Let's compare the storey displacements for both the structures for SPECX and SPECY.

\section{Storey Displacement}

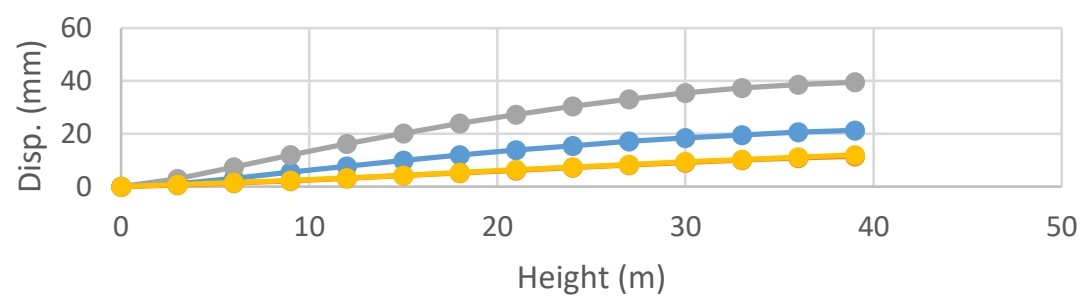

—Type A —Type B $\multimap$-Type A $\multimap$-Type B

\subsection{Time History Response}

Response spectrum plot for joint 18 (Type A) and Joint 22 (Type B) pseudo-spectral accelerations for time history ground acceleration compatible with IS1893 (Medium Soil).

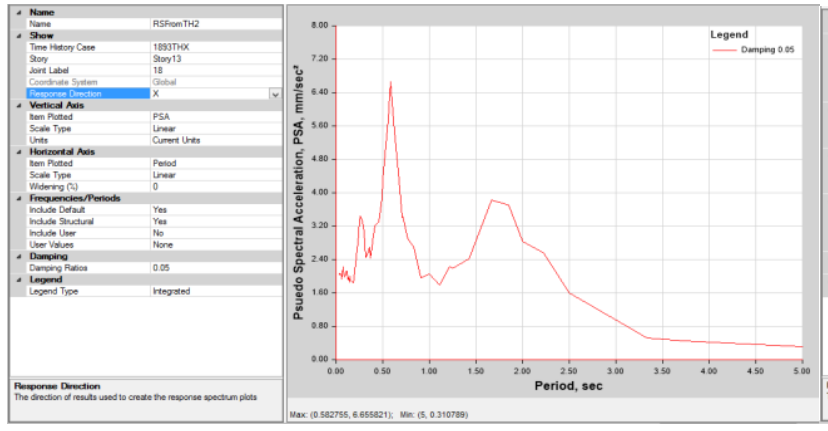

Type A (X)

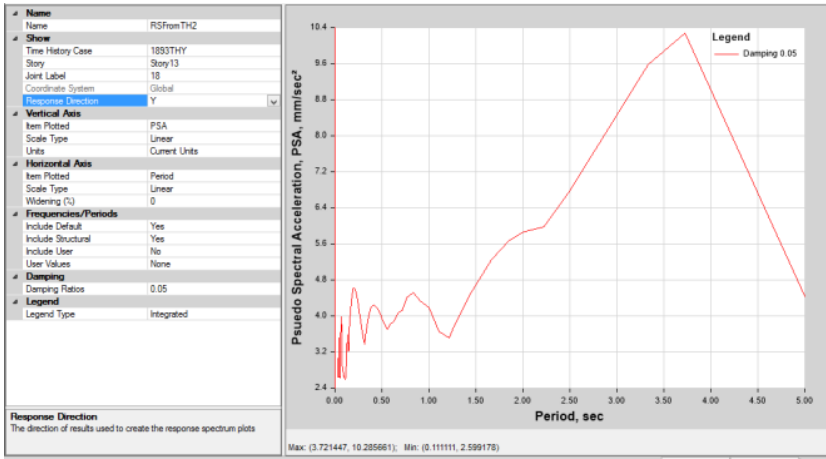

Type A (Y)

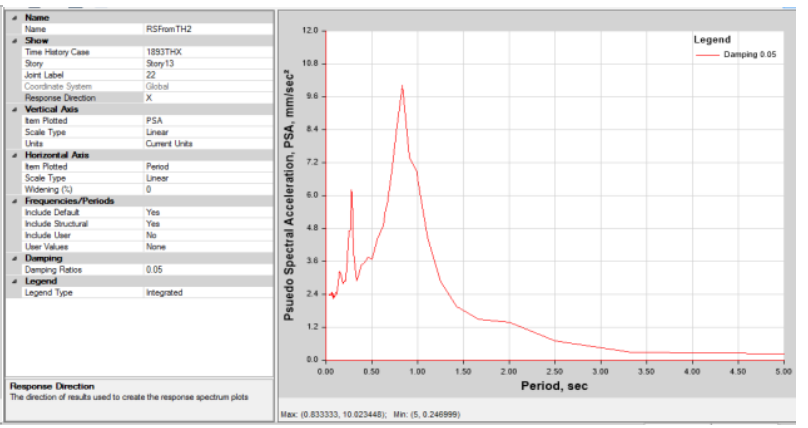

Type B (X)

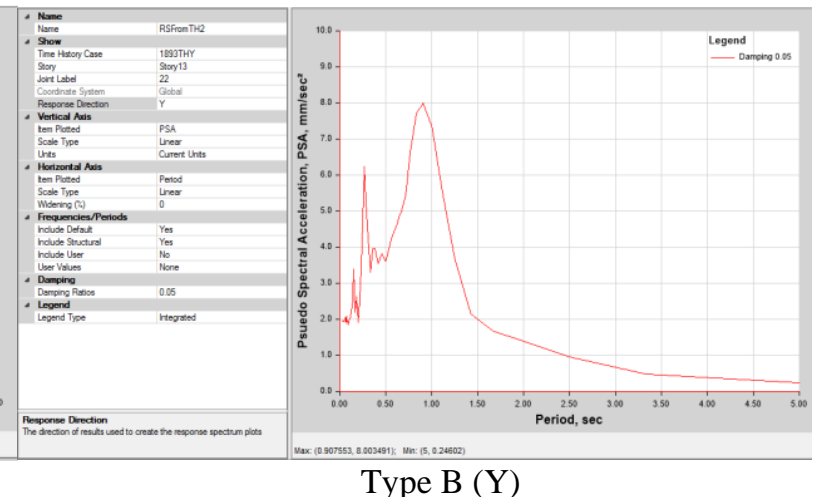


Response spectrum plot for joint 18 (Type A) and Joint 22 (Type B) pseudo-spectral accelerations for time history ground acceleration of Bhuj, Gujarat earthquake of 2001.

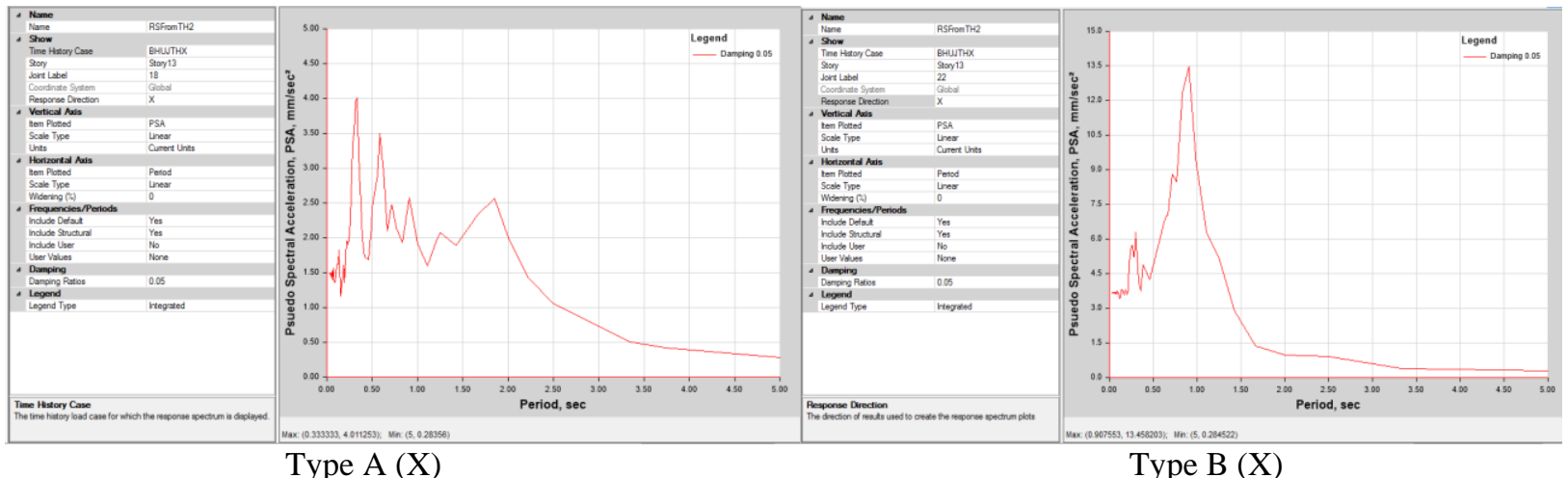

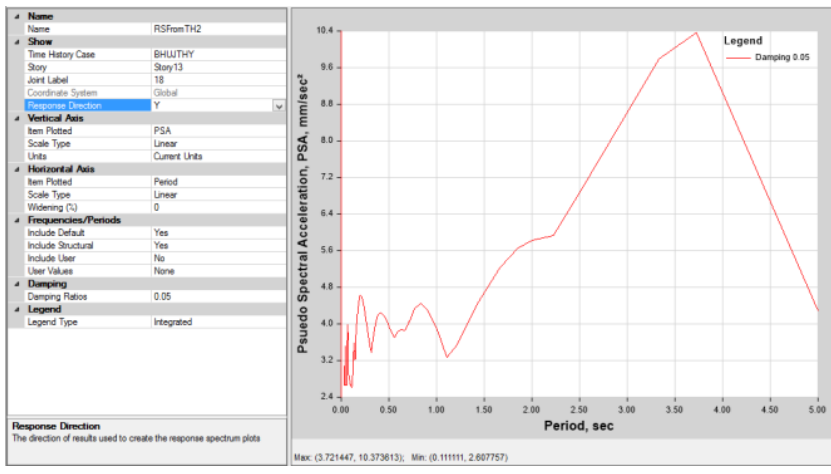

Type A (Y)

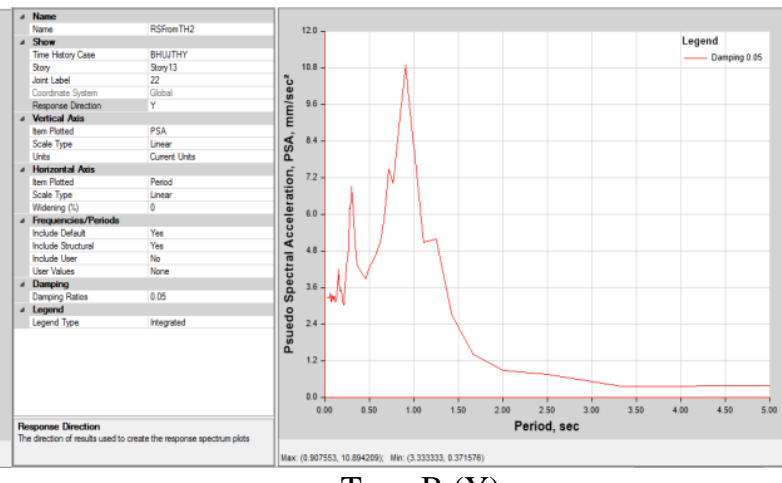

Type B (Y)

CONCLUSIONS

Time history examination was done on similar models for viable time history plot as IS1893 reaction range bend for medium soil. A similar analysis was reached out for time history of BHUJ, seismic tremor of 2001. Here, the elements change significantly. For instance, the base response for Type A structure for IS1893 time history is $139.44 \mathrm{kN}$ in X bearing just, which changes to $148.24 \mathrm{kN}$ in $\mathrm{X}$ and extra $18.04 \mathrm{kN}$ in Z heading (vertical) for Type B structure. Also, its further changes to $1000 \mathrm{kN}$ in $\mathrm{X}$ and extra $333.3 \mathrm{kN}$ in $\mathrm{Z}$ course for Type $\mathrm{C}$. The adjustment in Type $\mathrm{C}$ is a direct result of the suspicion of firmness boundaries of the Isolator being arbitrary. This would change, as explicit Isolator values are given in the model. While in the event of Type B, the corner to corner connect property allocated as Damper, is moving the shear at an edge to the base. This power is getting settled into two segments, as is the outcome.

\section{Basic Plane Frame of 10 Stories}

The elements of the structure's changes with the expansion of 6 additional accounts, however the essential variety of boundaries is pretty much the equivalent. True to form the removal decreases from $27 \mathrm{~mm}$ at top to $12 \mathrm{~mm}$ at top after incorporation of dampers. Additionally, the base response changes. Seen here the dislodging time history for Joint 92 at popular narrative of Type A (Bare Frame) is impressively higher than that of Joint 92 at popular narrative of Type B (With TMD) for IS1893 Time History. Comparative, conduct appeared for Bhuj quake time history.

We saw the separation of vibration at the base. While the whole structure uproots as an inflexible unit, the general dislodging of the individual stories is same as the uncovered casing structure. The thought for a base secluded structure to be effective is to make it unbending, which is essentially plausible for low stature structures. Subsequently, it is an ideal decision for institutional structures, business focuses, shopping centres and so forth.

The two pictures show the relocation time history for the quickening history of IS1893 and Bhuj seismic tremor. (Allude 4.9.1) Here we see, the genuine contrast in execution of the two structures. Joint 92 appeared by orange shading is for the straightforward structure, while Joint 95 appeared by green shading is the base detached structure. We can unmistakably observe the relocation is a lot higher for the base disconnected structure directly from the earliest starting point of the time history. However, this removal is a controlled one, so as to scatter the vitality leaving the structure and its occupants safe. 
The two pictures show the joint quickening for Type A and Type C outlines individually for speeding up time history of IS1893 and Bhuj quake. (Allude 4.9.1) We notice the base disconnected structure (appeared by cyan shading) gets practically immaterial increasing speed contrasted with the straightforward structure (appeared by red shading). This shows considerably less power for the base secluded structure and accordingly a more secure arrangement.

\section{Utilization of BTMD's}

A similar examination was additionally stretched out to full 3D models with the utilization of bidirectional tuned mass dampers and the outcomes got for seismic tremor powers in both $\mathrm{X}$ and $\mathrm{Y}$ headings. Here, one all the more structure model was presented with an alternate situation of dampers being presented in the structure. The story relocation for EQX was true to form, greatest for Type B followed by Type A, Type D and afterward Type C. Type D has lesser number of dampers than Type C. Comparable pattern could be seen for EQY, however the thing that matters is higher here. The story firmness is most extreme for Type B followed by Type D, Type an and Type C both for EQX and EQY.

In the uncommon instance of torsionally coupled structures, the story removal is exceptionally decreased for Type B and Type D true to form. However, strikingly here, the variety of position of TMD's isn't influencing the outcomes by any means. Truth be told, the uprooting bends consolidate. It was additionally seen that TMD's are powerful inside a scope of certain stature as it were. Expanding the mass of the damper makes the damper be viable, however we can build the mass just up to a specific breaking point. Generally, around $10 \%$ the mass of the primary structure utilized as damper is compelling. Presently, this can be practicable, for structures of medium stature without any problem. Be that as it may, for exceptionally tall structures, lifting such hefty mass to its ideal area is likewise a tough errand. This requires the requirement for additional modern techniques for diminishing the reaction of the structure for seismic burdens.

\section{REFERENCES}

[1] Kareem, A., Kijewski, Y, "Mitigation of Motions of Tall Buildings with Specific Examples of Recent Applications," Wind and Structures., Vol. 2, No. 3, pp. 201-251 (1999).

[2] Introduction to Structural Motion Control, Jerome J. Connor.

[3] Seismic Analysis of Structures, T K Datta, IIT Delhi.

[4] IS1893 (Part-1): 2016 'Criteria for Earthquake Resistant Design of Structures'.

[5] IS 875 (Part-3): 2015 'Design Loads (Other than Earthquake) for Buildings and Structures, Part-3 - Wind Load'.

[6] Taylor, D.P. and M.C. Constantinou. (1996), Fluid Dampers for Applications of Seismic Energy Dissipation and Seismic Isolation, Publication of Taylor Devices, Inc., 1996.

[7] Tamura, Y. (1997), Application of Damping Devices to Suppress WindInduced Responses of Buildings, Proceedings of the 2nd European and African Conference on Wind Engineering, Palazzo Ducale, Genova, Italy, 1:45-60.

[8] Sakamoto, M. (9-10 Dec. 1993), Practical Applications of Active Structural Response Control and Earthquake \&Strong Wind Observation Systems, Planning Workshop for the Hong Kong International Full-Scale Control TestFacility, Hong Kong University of Science \& Technology.

[9] Sakamoto, M. and Kobori, T. (Dec. 1996), Applications of Structural Response Control (Reviews from the Past and Issues Toward the Future), Proceedings of the Second International Workshop on Structural Control, Hong Kong.

[10] Kareem, A., (1983), Mitigation of Wind Induced Motion of Tall Buildings, Journal of Wind Engineering and Industrial Aerodynamics, 11(1-3): 273284.

[11] Kareem, A. (1990), Reduction of Wind Induced Motion Utilizing a Tuned Sloshing, Journal of Wind Engineering and Industrial Aerodynamics, 36: 72537.

[12] Kareem, A. (27-30 June 1993), Tuned Liquid Dampers: Past, Present, and

[13] Future, Proceedings of the 7th U.S. National Conference on Wind Engineering.

[14] Kareem, A., and M. Tognarelli. (Oct. 1994), Passive \& Hybrid Tuned Liquid Dampers, Structural Engineering Forum: 26-30.

[15] Symans, M.D. and M.C. Constantiou. (1996), Experimental Study of Seismic Response of Structures with Semi-Active Damping Control Systems, Proceedings of Structures Congress X.IV, Chicago, April 15-18. 